\title{
SCATTERING FOR SOLUTIONS OF NLS IN THE EXTERIOR OF A 2D STAR-SHAPED OBSTACLE
}

\author{
Fabrice Planchon and Luis Vega
}

\begin{abstract}
We prove that solutions to non-linear Schrödinger equations in two dimensions and in the exterior of a bounded and smooth star-shaped obstacle scatter in the energy space. The non-linear potential is defocusing and grows at least as the quintic power.
\end{abstract}

\section{Introduction and main results}

In this paper, we are interested in the initial value problem

$$
\left\{\begin{array}{l}
i \partial_{t} u+\Delta u=\epsilon|u|^{p-1} u \quad u \in \mathbb{R}^{2} \backslash \Sigma=\Omega, \quad \epsilon \in\{0,1\}, \quad p \geq 5, \\
\left.u\right|_{\partial \Sigma}=0 \\
u(x, 0)=u_{0}(x) \in H_{0}^{1}(\Omega)
\end{array}\right.
$$

and more particularly in the proof of scattering properties of the solutions. With respect to $\Sigma$ we will assume that it is a star-shaped domain contained in a compact set $K$, and that its boundary $\partial \Sigma=\partial \Omega$ is a smooth curve in $\mathbb{R}^{2}$.

As it is well-known dimensions one and two are the most delicate ones regarding scattering questions even if no obstacle is considered and one works in the full space $\mathbb{R}^{d}$. The main obstruction comes from the sign of the bilaplacian term that appears in the classical Morawetz-Lin-Strauss inequality [9]. This sign turns out to be the wrong one for $d=1,2$. In dimensions three and higher and in $\mathbb{R}^{d}$ the question was settled in the energy space by Ginibre and Velo in [6]. The obstruction in the dimension was finally removed, still in full space, by Nakanishi [10], but his technique does not seem directly applicable in the exterior of a two-dimensional (2D) domain; that is our interest here. One should remark that the 1D case (with both Dirichlet or Neuman boundary conditions) follows directly from the $\mathbb{R}$ case, as symmetry with respect to $x=0$ is preserved by the non-linear flow. Hence for exterior domains, the $2 \mathrm{D}$ case may be seen as the most difficult one.

Another fundamental contribution regarding global existence and scattering was the introduction by Colliander et al. in $[4,5]$ of interactive Morawetz inequalities. However a similar obstruction in low dimensions appears as well. Again the difficulty comes from the sign of the bilaplacian term that appears in the use of the bilinear multiplier. It turns out that this obstruction can be easily bypassed (see $[2,11]$ ) and as a consequence the scattering of non-linear Schrödinger (NLS) equations for $L^{2}$

Received by the editors November 4, 2011.

2000 Mathematics Subject Classification. 35255.

The first author was partially supported by A.N.R. grant SWAP.

The second author was partially supported by UPV/EHU and by MEC grant MTM 2007-03029. 
subcritical non-linearities is by now fairly well understood (see for example [7] for a good survey, and also [3] for earlier results in 1D using a quadrilinear interactive Morawetz inequality).

In [11] the bilinear multiplier technique is also used in the setting of exterior domains with Dirichlet boundary conditions. In order to explain our results, we have to introduce some notation.

Let

$$
n \geq 1, \quad p>1, \quad \epsilon \in\{-1,0,1\}, \quad \Omega_{1}, \Omega_{2} \subset \mathbb{R}^{n},
$$

with smooth boundaries $\partial \Omega_{1}, \partial \Omega_{2}$, and $u_{1}, u_{2}$ solutions of

$$
\mathrm{i} \partial_{t} u_{j}+\Delta u_{j}=\epsilon\left|u_{j}\right|^{p-1} u_{j}, \quad \text { with }\left.u_{j}\right|_{\partial \Omega_{j}}=0 \quad j=1,2 .
$$

Denote for $j=1,2$

$$
M\left(u_{j}\right)=\int_{\Omega_{j}}\left|u_{j}\right|^{2} d x ; \quad E\left(u_{j}\right)=\frac{1}{2} \int_{\Omega_{j}}|\nabla u|^{2}+\frac{\epsilon}{p+1} \int_{\Omega_{j}}\left|u_{j}\right|^{p+1} d x,
$$

the mass and energy that are conserved quantities. As in [11] we shall use the Radon transform

$$
R(f)(s, \omega)=\int_{\{x \cdot \omega=s\} \cap \Omega} f d \mu_{s, \omega},
$$

with $\Omega$ either $\Omega_{1}$ or $\Omega_{2}$. In other words, we extend $f$ as zero outside of $\Omega$ and compute its Radon transform.

We set

$$
I_{\rho}(t)=I_{\rho}\left(u_{1}(t), u_{2}(t)\right)=\int_{\Omega_{1} \times \Omega_{2}} \rho(x-y)\left|u_{1}(x)\right|^{2}\left|u_{2}(y)\right|^{2} d x d y .
$$

Then a simple modification of the proof of Theorem 2.5 in [11] gives us ${ }^{1}$

Theorem 1. Let $\omega \in \mathbb{R}^{n}, n>1$, with $|\omega|=1$ and $\rho_{\omega}(z)=|z \cdot \omega|, u_{j}$ solution of (1.2) with $j=1,2$. Then if $x=x^{\perp}+s \omega$ and $x^{\perp} \cdot \omega=0$, we have

$$
\begin{aligned}
\frac{d^{2}}{d t^{2}} I_{\rho_{\omega}}= & \int_{s}\left|\partial_{s}\left(\mathcal{R}\left(u_{1} \bar{u}_{2}\right)\right)\right|^{2} d s \\
& +\epsilon \frac{p-1}{p+1}\left(\int_{s} \mathcal{R}\left(\left|u_{1}\right|^{2}\right) \mathcal{R}\left(\left|u_{2}\right|^{p+1}\right) d s+\int_{s} \mathcal{R}\left(\left|u_{2}\right|^{2}\right) \mathcal{R}\left(\left|u_{1}\right|^{p+1}\right) d s\right) \\
& +\int_{s} \int_{x \cdot \omega=s} \int_{y \cdot \omega=s} \mid u_{1}\left(x^{\perp}+s \omega\right) \partial_{s} u_{2}\left(y^{\perp}+s \omega\right) \\
& -\left.u_{2}\left(y^{\perp}+s \omega\right) \partial_{s} u_{1}\left(x^{\perp}+s \omega\right)\right|^{2} d x^{\perp} d y^{\perp} d s \\
& -\int_{\partial \Omega_{1} \times \Omega_{2}}\left|u_{2}\right|^{2}(y) \partial_{n} \rho_{\omega}(x-y)\left|\partial_{n} u_{1}\right|^{2}(x) d S_{x} d y \\
& -\int_{\Omega_{1} \times \partial \Omega_{2}}\left|u_{1}\right|^{2}(x) \partial_{n} \rho_{\omega}(x-y)\left|\partial_{n} u_{2}\right|^{2}(y) d x d S_{y},
\end{aligned}
$$

where $\partial_{n}$ is the outgoing normal vector field on $\partial \Omega$.

\footnotetext{
${ }^{1}$ There are some misprints in the second and fourth term of the right-hand side of (2.19) in [11]. A factor 2 is missing in both of them.
} 
A simple look at the statement of the above theorem tells us that it is useless unless the boundary terms appearing in (1.6) are under control. In [11] this is done using the so-called local smoothing property that follows from a variation of Morawetz's multiplier. As a consequence a sufficient geometric condition on the boundary naturally appears: if $u$ is a solution to (1.2), and $n(x)$ denotes the outgoing normal vector to $\partial \Omega$ at $x$, local smoothing for the non-linear solution $u$ is obtained provided

$$
\int_{0}^{T} \int_{\partial \Omega}(x \cdot n(x))\left|\partial_{n} u\right|^{2} d S_{x} d t \geq 0 .
$$

In particular, it is sufficient to assume that $\Omega$ is the complement of a star-shaped domain and this is what we are going to assume in this paper. Unfortunately the use of Morawetz's multiplier introduces again the restriction on the dimension and therefore the result in [11] (Proposition 2.7) is given in dimension three and higher. In this paper, we remove such a restriction.

The main new idea is to use Theorem 1 with $\Omega=\Omega_{1}=-\Omega_{2}, u_{1}=u$ and $u_{2}(x)=$ $u(-x)$ and $u$ a solution of (1.1). Equivalently we may consider $\widetilde{\rho}=\rho(x+y)$ instead of $\rho(x-y)$ in the definition of $I_{\rho}$ in (1.5). We then sum up the new (with $\widetilde{\rho}_{\omega}$ ) and old (with $\rho_{\omega}$ ) estimates: it follows that some cancellation occurs between boundary terms of (1.6), and integrating over $\omega \in \mathbb{S}^{n-1}$ one obtains that the boundary term is bounded above by

$$
\int_{\partial \Omega \times \Omega}\left|\partial_{n} u(x)\right|^{2}|u(y)|^{2}\langle y\rangle^{-1} d S_{x} d y
$$

where the gain in the above inequality comes from the weight $\langle y\rangle=\left(1+|y|^{2}\right)^{1 / 2}$.

It turns out this gain is sufficient. The reason is that we can use the tensor product technique developed in $[2,3]$. In order to do that we construct $v(x, y)=u(x) u(y)$, solution of NLS in $\Omega \times \Omega$, and use the local smoothing inequality obtained from Morawetz's multipliers in dimension $n=4$; see Proposition 1 below. That fits perfectly well with our purposes.

Remark 1. In the $\mathbb{R}^{n}$ case, all monotonicity formulae stemming from virial identities are related to the conservation of the momentum, a key property which is lost on domains. The boundary terms then appear as a direct consequence. The star-shaped case provides a class of obstacles for which this loss is controllable by an integration by parts argument (the usual virial in dimension $n \geq 3$ or the tensorialized one in the present paper for $n=2$ ). For the linear equation, microlocal techniques allow to control the momentum through local smoothing estimates - as they control boundary terms, see [11] -, when the exterior domain is non-trapping (light rays escape to spatial infinity). But extending such a control to the non-linear setting appears a challenging task.

We denote by $H_{0}^{1}=H_{0}^{1}(\Omega)$ the energy space, which is also the domain of the square root of $-\Delta_{\Omega}$, where $\Delta_{\Omega}$ is the Dirichlet Laplacian on $\Omega$. We are now able to state our main theorem. 
Theorem 2. Let $\Omega$ be $\mathbb{R}^{2} \backslash \Sigma$, where $\Sigma$ is a star-shaped and bounded domain, and $u_{0} \in H_{0}^{1}(\Omega)$. Then, there exits a unique solution of

$$
\left\{\begin{array}{l}
i \partial_{t} u+\Delta u=|u|^{p-1} u \quad x \in \Omega, \quad t \in \mathbb{R} \quad p \geq 5 \\
\left.u\right|_{\partial \Omega}=0 \\
u(x, 0)=u_{0}(x)
\end{array}\right.
$$

such that

$$
u \in \mathcal{C}\left(\mathbb{R}: H_{0}^{1}(\Omega)\right) \cap L_{t}^{p-1} L_{x}^{\infty} .
$$

Moreover, there exist unique $u_{+}, u_{-} \in H_{0}^{1}(\Omega)$ such that

$$
\left\|u(\cdot, t)-e^{i t \Delta_{\Omega}} u_{ \pm}\right\|=o(1) \quad t \rightarrow \pm \infty .
$$

Here $e^{i t \Delta_{\Omega}}$ is the solution of the linear problem (i.e., (1.1) with $\epsilon=0$ ).

The reader can guess that the key property of the solution obtained in the above theorem is that it satisfies the global in time Strichartz estimate $L_{t}^{p-1} L_{x}^{\infty}$. The key step in that direction stems from Theorem 1 and the local smoothing estimate that we mentioned before: one gets that $D^{1 / 2}\left(|u|^{2}\right)$ is in $L^{2}$ of both variables space and time. This is also true for linear solutions. This turns out to be the last key ingredient for the proof of Theorem 2. We have the following result.

Theorem 3. Let $\Omega=\mathbb{R}^{2} \backslash \Sigma$, where $\Sigma$ is star-shaped and bounded. Define $e^{i t \Delta_{\Omega}} u_{0}$ as the solution of

$$
\left\{\begin{array}{l}
i \partial_{t} u+\Delta u=0 \quad x \in \Omega, \quad t \in \mathbb{R} \\
\left.u\right|_{\partial \Omega}=0 \\
u(x, 0)=u_{0}(x)
\end{array}\right.
$$

Then

$$
\left\|D^{1 / 2}\left(|u|^{2}\right)\right\|_{L_{t}^{2} L_{x}^{2}} \leq C\left\|u_{0}\right\|_{L^{2}}^{3 / 2}\left\|u_{0}\right\|_{H_{0}^{1}}^{1 / 2}
$$

and, for $0 \leq s \leq 3 / 4$,

$$
\left\|D^{s} u\right\|_{L_{t}^{4} L_{x}^{8}} \leq C\left\|u_{0}\right\|_{H_{0}^{s+1 / 4}}
$$

As a consequence

$$
\|u\|_{L_{t}^{4} L_{x}^{\infty}} \leq C\left\|u_{0}\right\|_{H_{0}^{1}}
$$

Here $D^{s}=(-\Delta)^{s / 2}$ and $H_{0}^{s}(\Omega)=H_{0}^{s}$ denotes the domain of the operator $\left(-\Delta_{\Omega}\right)^{s / 2}$ given by the spectral theorem applied to the Dirichlet Laplacian on $\Omega$.

Remark 2. Notice that under the smoothness conditions on $\Omega, D^{s}\left(-\Delta_{\Omega}\right)^{-s / 2}$ is bounded in $L^{p} \quad 1<p<\infty$. See for example [8] and references therein, for the proof of this fact.

Remark 3. One may extend Theorem 2 to non-linearities with $p<5$, by using the known range of Strichartz estimates outside non-trapping obstacles from [1]: for $\varepsilon>0$,

$$
\|u\|_{L_{t}^{\frac{3}{1-\varepsilon}} L_{x}^{\frac{2}{\varepsilon}}} \leq C(\varepsilon)\left\|u_{0}\right\|_{H_{0}^{\frac{1}{3}(1-\varepsilon)}} .
$$


This allows us to replace $p=4$ in Theorem 3 by $p=3 /(1-\varepsilon)$, which will however not fill the expected range $3<p<5$ in Theorem 2 , by analogy with the $\mathbb{R}^{2}$ case, but rather provide $p>4$. We elected to keep the argument mostly self-contained, as the linear estimates from Theorem 3 are enough for our purposes in the range $p \geq 5$.

In the next section, we prove Theorems 2 and 3 and give the necessary propositions to be able to use Theorem 1. This one is proved as Theorem 2.5 in [11] and therefore we omit the details.

\section{Proofs}

We will first prove Theorem 3. We need some lemmas.

Lemma 1. For $\omega \in \mathcal{S}^{1}$ define $\rho_{\omega}(x)=|x \cdot \omega|$ and

$$
A_{\omega}=\{(x, y) \in \partial \Omega \times \Omega:|y \cdot \omega| \leq|x \cdot \omega|\} .
$$

Then if $y \in \Omega$

$$
\begin{aligned}
& \left\{(x, y) \in \partial \Omega \times \Omega: \partial_{n} \rho_{\omega}(x-y)+\partial_{n} \rho_{\omega}(x+y) \neq 0\right\} \\
& \subset\{\operatorname{sig}(x-y) \cdot \omega+\operatorname{sig}(x+y) \cdot \omega \neq 0\}=A_{\omega},
\end{aligned}
$$

and

$$
\sup _{x \in \partial \Omega}\left|\int_{\mathcal{S}^{1}} \chi_{A_{\omega}} d \sigma(\omega)\right| \leq \frac{C}{\langle y\rangle},
$$

Proof. We can assume without loss of generality that $\omega=(1,0)$. Then if $x=\left(x_{1}, x_{2}\right)$, $y=\left(y_{1}, y_{2}\right)$ we have that

$$
\begin{aligned}
\partial_{n} \rho_{\omega}(x-y)+\partial_{n} \rho_{\omega}(x+y) & =\operatorname{sig}(x-y) \cdot \omega+\operatorname{sig}(x+y) \cdot \omega \\
& \left.\left.=\operatorname{sig}\left(x_{1}-y_{1}\right)\right)+\operatorname{sig}\left(x_{1}+y_{1}\right)\right),
\end{aligned}
$$

and hence by inspection we get $(2.1)$

As for (2.2) we have that if $|y \cdot \omega|<|x \cdot \omega|$ then $|y||\cos (y, \omega)| \leq \operatorname{diam}(\partial \Omega)$. Thus, for $|y|$ large enough

$$
|y|\left|\theta_{y}-\theta_{\omega} \pm \frac{\pi}{2}\right| \leq M
$$

with $\frac{y}{|y|}=\left(\cos \theta_{y}, \sin \theta_{y}\right)$ and $\omega=\left(\cos \theta_{\omega}, \sin \theta_{\omega}\right)$. The lemma easily follows.

Proposition 1. Let $\Omega$ be $\mathbb{R}^{2} \backslash \Sigma$, with $\Sigma$ a bounded smooth star-shaped obstacle. Assume $u$ is a solution of

$$
\begin{aligned}
i \partial_{t} u+\Delta u & =\epsilon|u|^{p-1} u, \quad p>1 \\
\left.u\right|_{\partial \Omega} & =0
\end{aligned}
$$

with $\epsilon \in\{0,1\}$. Then

$$
\int_{\mathbb{R}} \int_{\partial \Omega \times \Omega}\left|\partial_{n} u(x)\right|^{2}|u(y)|^{2} d S_{x} \frac{d y}{\langle y\rangle} d t \leq C M^{3 / 2} E^{1 / 2}
$$

with $M$ and $E$ as in (1.3). 
Proof. Define $v(x, y)=u(x) u(y)$ solution of the problem

$$
\begin{aligned}
\mathrm{i} \partial_{t} v+\Delta v & =\epsilon\left(|u|^{p-1}(x)+u^{p-1}(y)\right) v, \quad(x, y) \in \Omega \times \Omega, \\
\left.v\right|_{\partial(\Omega \times \Omega)} & =0 \\
v(x, y, 0) & =u_{0}(x) u_{0}(y) .
\end{aligned}
$$

Then, consider for $h(x, y)=\sqrt{|x|^{2}+|y|^{2}}$

$$
M_{h}(t)=\int_{\Omega \times \Omega}|v|^{2}(x, y, t) h(x, y) d x d y
$$

and compute $\frac{d^{2}}{d t^{2}} M_{h}(t)$. The details can be found for example in [11] (p. 278), up to easy modifications to handle the non-linear term in (2.4). We just have to bother about $\partial_{n} h$ with $n$ the normal to $\partial(\Omega \times \Omega)$, namely

$$
\begin{array}{ll}
n=\left(n_{x}, 0\right), & \text { if } \quad x \in \partial \Omega, \quad y \in \Omega, \\
n=\left(0, n_{y}\right), & \text { if } \quad x \in \partial \Omega, \quad y \in \Omega .
\end{array}
$$

Hence

$$
\partial_{n} h(x, y)=\frac{n_{x} \cdot x}{\sqrt{|x|^{2}+|y|^{2}}}
$$

if $(x, y) \in \partial \Omega \times \Omega$ and similarly in the other case. We recall that the kernel of a starshaped domain is the set of points with respect to which the domain is star-shaped. If we assume that the kernel of our obstacle contains a disc (note that the kernel is always convex) then we may exclude the situation where $n_{x} \cdot x=0$, by averaging over this disk if necessary. Abusing notation by forgetting about this possible average over the base point, we then get that

$$
\left|\partial_{n} h(x, y)\right| \geq \frac{C}{|y|}
$$

with $C>0$. The argument follows as in p. 278 of [11] because

$$
\left|\partial_{n} v\right|^{2}=\left|\partial_{n} u(x)\right|^{2}|u(y)|^{2},
$$

if $(x, y) \in \partial \Omega \times \Omega$ and a similar expression if $(x, y) \in \Omega \times \partial \Omega$.

If the kernel of the obstacle is a single point (or even a segment), we need to address the situation where there exist points $x \in \partial \Omega$ such that $n_{x} \cdot x=0$. We may repeat the computation of [11] with weight $\rho(x, y)=\sqrt{1+|x|^{2}+|y|^{2}}$, but the desired control on the boundary does not follow by a subsequent computation with a weight like the distance to the boundary, because in our setting the $4 \mathrm{D}$ obstacle is not compact and we need to account for the trace of $\partial_{n} h$ on the boundary. Let us summarize what the computation with weight $\rho$ provides, following [11]:

Lemma 2. Under the conditions of Proposition 1,

$$
\int_{\mathbb{R}} \int_{\Omega \times \Omega} \frac{|\not \nabla v|^{2}}{\rho(x, y)}+\frac{|\nabla v|^{2}+|v|^{2}}{\rho^{3}(x, y)}+\frac{\left(|u|^{p-1}(x)+|u|^{p-1}(y)\right)|v|^{2}}{\rho(x, y)} d x d y \leq C M^{3 / 2} E^{1 / 2},
$$

where $\not$ denotes the (4D) angular gradient. 
We now proceed with the remaining case in the proof of Proposition 1: control of the boundary term in the situation where there exist points $x \in \partial \Omega$ such that $x \cdot n(x)=0$. While Lemma 2 is not enough to conclude directly, it will be a key ingredient in what follows. Recall we aim at controlling the left-hand side of (2.3).

Let $R$ be such that $\partial \Omega \subset B(0, R-1)$. For the part of the $4 \mathrm{D}$ boundary $(\partial \Omega \cap$ $B(0, R))^{2}$ we may proceed as in [11], using the control of $\nabla v$ in $(2.5)$, because the weight $\rho^{3}$ is irrelevant. The remaining part of the $4 \mathrm{D}$ boundary is a union of cylinders $\partial \Omega \times\left(\mathbb{R}^{2} \backslash B(0, R)\right)$ and $\left(\mathbb{R}^{2} \backslash B(0, R)\right) \times \partial \Omega$. They will be treated similarly and we restrict our attention to the first one (boundary in $x$ and exterior of a large ball in $y$ ). We may further divide the $y$-region in a finite number of angular sectors: without loss of generality we now assume to be in such a sector $S=\left\{\left|y_{2}\right|<\left|y_{1}\right| / 10\right\}$. Notice how being outside a ball forces $\left|y_{1}\right|>R / 2$.

From the compactness of $\partial \Omega$ and its smoothness as a curve in $\mathbb{R}^{2}$, there exists a vector field $Z$ such that $Z$ is defined in $\Omega$, its restriction on $\partial \Omega$ is the normal derivative, and it vanishes outside $B(0, R)$. In a coordinate system,

$$
Z=a(x) \partial_{x_{1}}+b(x) \partial_{x_{2}}
$$

where $a(x), b(x)$ are smooth functions vanishing for $|x|>R$. We define two 4D vector fields on our $4 \mathrm{D}$ region of interest, $(\Omega \backslash B(0, R)) \times S$ :

$$
Y_{k}=y_{1} \partial_{x_{k}}-x_{k} \partial_{y_{1}} \text {, with } k=1,2 .
$$

Notice that $Y_{1}$ and $Y_{2}$ are, up to a factor $\left(\sqrt{|x|^{2}+|y|^{2}}\right)^{-1}$, angular derivatives (in 4D). We may now define

$$
Y=\alpha(x, y) Y_{1}+\beta(x, y) Y_{2}
$$

where the support of $\alpha$ and $\beta$ is in $(\Omega \backslash B(0, R)) \times S$ and their respective value is adjusted such that $(Y v)_{\mid x \in \partial \Omega} \approx h^{-1}(x) Z v$, as $\partial_{y_{1}} v=0$ on $\partial \Omega \times \Omega$ : set $\alpha(x, y)=$ $\phi(y /|y|) a(x) / y_{1}^{2}$ and $\beta(x, y)=\phi(y /|y|) b(x) / y_{1}^{2}$ where $\phi(y /|y|)$ is the identity in the angular sector $S$, and vanishes outside $2 S$. One should think of the following computation as an analog of the one in [11] p. 278/279, but where we think of the region of $4 \mathrm{D}$ space we are integrating over as being foliated by spheres $|x|^{2}+|y|^{2}=C$ rather than planes $|y|=C$. We use the following momentum

$$
J=2 \operatorname{Im} \int_{\Omega \times \Omega} \bar{v}(Y v) d x d y .
$$

For the sake of notational convenience, we set $X=(x, y)$ and $Y=A(X) \cdot \nabla_{X}=A \cdot \nabla$ and $N(u)=|u|^{p-1}(x)+|u|^{p-1}(y)$. The following computation is standard, does not depend on the specific form of $A$ and similar to the one in [11] where $A=\nabla h$;

$$
\begin{aligned}
\partial_{t}\left(2 \operatorname{Im} \int \bar{v} \nabla v \cdot A\right)= & -2 \operatorname{Im} \int \partial_{t} v(2 \nabla \bar{v} \cdot A+\bar{v} \nabla \cdot A) \\
= & -4 \operatorname{Re} \int \Delta v \nabla \bar{v} \cdot A+2 \int|\nabla v|^{2} \nabla \cdot A-\int|v|^{2} \Delta \nabla \cdot A \\
& +2 \int N \nabla\left(|v|^{2}\right) \cdot A+|v|^{2} N \nabla \cdot A .
\end{aligned}
$$


We have then (with $A_{n}$ the normal component of $A$ on the boundary and $d \sigma$ the surface measure on it, with orientation toward the domain)

$$
\begin{aligned}
2 \operatorname{Re} \int \Delta v \nabla \bar{v} \cdot A= & -2 \int\left|\partial_{n} v\right|^{2} A_{n} d \sigma-2 \operatorname{Re} \int \nabla v \cdot \nabla(A \cdot \nabla \bar{v}) \\
= & -2 \int\left|\partial_{n} v\right|^{2} A_{n} d \sigma-2 \operatorname{Re} \int A \cdot \nabla(\nabla \bar{v} \cdot \nabla v) \\
& +2 \operatorname{Re} \int[A \cdot \nabla, \nabla] \bar{v} \cdot \nabla v
\end{aligned}
$$

and by substitution and integration by parts in the middle term and the non-linear term above,

$$
\begin{aligned}
\partial_{t}\left(2 \operatorname{Im} \int \bar{v} \nabla v \cdot A\right)= & 2 \int\left|\partial_{n} v\right|^{2} A_{n} d \sigma-4 \operatorname{Re} \int[A \cdot \nabla, \nabla] \bar{v} \cdot \nabla v \\
& -\int|v|^{2} \Delta \nabla \cdot A+2 \frac{p-1}{p+1} \int|v|^{2}\left(|u|^{p-1}(x) \nabla_{x} \cdot A_{x}\right. \\
& \left.+|u|^{p-1}(y) \nabla_{y} \cdot A_{y}\right),
\end{aligned}
$$

where $A_{x}$ (resp. $A_{y}$ ) stands for the projection over the $x$ coordinates (resp. the $y$ coordinates) of the vector field $A$. Given our choice of $Y=A \cdot \nabla$, the boundary term is what we seek, up to an harmless factor $h(x, y) / y_{1}$ :

$$
\int\left|\partial_{n} v\right|^{2} A_{n} d \sigma=\int Y v \nabla \bar{v} d \sigma=\int_{\partial \Omega \times \Omega} \frac{\phi(y /|y|)}{y_{1}}\left|\partial_{n} v\right|^{2} d S_{x} d y .
$$

The term $N(u)|v|^{2}$ will be under control by Lemma 2. For the $|v|^{2}$ term, we may use Poincaré's inequality on the spheres $|x|^{2}+|y|^{2}=C$ to control it as well by $\int \rho^{-1}(x, y)|\not \nabla v|^{2}$ (notice how when deriving in $x$ we do not gain decay in the coefficients of $Y)$.

We are left with the term carrying $[Y, \nabla]$ : for any scalar function $\gamma$, we have $\left[\gamma Y_{k}, \partial\right]=\gamma\left[Y_{k}, \partial\right]-(\partial \gamma) Y_{k}=-(\partial \gamma) Y_{k}$; hence the vector field $[Y, \nabla]$ is spanned by angular derivatives. Moreover, $|\partial \gamma| \lesssim|\gamma|$ (as the worst situation is when the derivative would hit $a(x)$ or $b(x)$, which does not gain decay in $y)$ and $\gamma \approx y_{1} / \rho(x, y)$, so that

$$
\int|[Y, \nabla] \bar{v} \cdot \nabla v| \lesssim \int \frac{|\not \nabla v|^{2}}{\rho(x, y)}
$$

which is controlled by Lemma 2 after time integration. This concludes the proof of Proposition 1.

Proposition 2. Under the conditions of Proposition 1

$$
\left.\left.\int_{\mathbb{R}} \int_{\Omega \times \Omega}\left|D^{1 / 2}\right| u\right|^{2}\right|^{2} d x d y \leq C M^{3 / 2} E^{1 / 2}
$$

Proof. We use Theorem 1 with $u_{1}=u(x)$ and $u_{2}=u(-x), \Omega^{\prime}=-\Omega$. Then, if $y \in \Omega^{\prime}$

$$
\partial_{n} \rho_{\omega}(x-y)=\partial_{n} \rho_{\omega}(x+y) \text {. }
$$


We use again Theorem 1 with $u=u_{1}=u_{2}$ and $\Omega_{1}=\Omega_{2}=\Omega$. Then we sum the two identities and integrate in time once. After discarding some positive terms we get

$$
\begin{aligned}
& \left.\left.\int_{0}^{T} \int_{s}\left|\partial_{s} \mathcal{R}\right| u\right|^{2}(s, \omega)\right|^{2} d s d \omega \\
& \quad-2 \int_{\partial \Omega \times \Omega}\left|\partial_{n} u(x)\right|^{2}|u(y)|^{2}\left(\partial_{n} \rho_{\omega}(x-y)+\partial_{n} \rho_{\omega}(x+y)\right) d S_{x} d y \\
& \quad-2 \int_{\Omega \times \partial \Omega}\left|\partial_{n} u(y)\right|^{2}|u(x)|^{2}\left(\partial_{n} \rho_{\omega}(x-y)+\partial_{n} \rho_{\omega}(x+y)\right) d x d S_{y} \\
& \quad \leq C M^{3 / 2} E^{1 / 2}
\end{aligned}
$$

where the right-hand side follows from the trivial bounds of $I_{\rho_{\omega}}^{\prime}$.

We conclude the argument integrating on $\omega \in \mathbb{S}^{1}$ and using the well-known Plancherel's Theorem for the Radon transform as in [11], (Prop. 2.2) to obtain

$$
\left\|D^{1 / 2}\left(|u|^{2}\right)\right\|_{L_{x,[0, T]}^{2}} .
$$

For the boundary term, we use Lemma 1 and then Proposition 1 . Note that both lemmas are symmetric in the $(x, y)$ variables.

Remark 4. The astute reader will have noticed by now that the entire computation may be performed directly, with the (integrated over $\omega$ ) weight $\rho(x, y)=|x-y|+|x+y|$. We feel the directional weight to be more natural, with the key Lemma 1 having a simple geometrical proof. In fact, we were led to the present result from considering simple obstacles with symmetries (e.g., the disc).

Proposition 3. Under the conditions of Lemma 1 and $\epsilon=0$,

$$
\left\|D^{s} u\right\|_{L_{t}^{4} L_{x}^{8}} \leq C\left\|u_{0}\right\|_{H_{0}^{s+1 / 4}}
$$

Proof. Take $u_{0}$ a frequency localized function (according to the Dirichlet Laplacian of $\Omega$ ). Then (2.7) is a consequence of (2.6) and Sobolev embedding. The general case follows by the square function estimates on domains and the boundedness in $L^{p}$, $1<p<\infty$, of the corresponding Riesz transforms, see for example [8], Theorem 1.2 and Remark (1.10).

Note that Propositions 2 and 3 imply Theorem 3. We already have all the necessary ingredients for the proof of Theorem 2 .

Proof of Theorem 2. For simplicity, we will consider the particular case $p=5$. The necessary changes needed for the rest of the powers will be outlined at the end of the proof.

We first notice that by Gagliardo-Nirenberg inequality

$$
\|f\|_{L^{\infty}\left(\mathbb{R}^{2}\right)} \leq C\|f\|_{L^{8}\left(\mathbb{R}^{2}\right)}^{2 / 3}\left\|D^{3 / 4} f\right\|_{L^{8}\left(\mathbb{R}^{2}\right)}^{1 / 3} .
$$

Hence

$$
\left\|\mathrm{e}^{\mathrm{i} t \Delta_{\Omega}} u_{0}\right\|_{L_{t}^{4} L_{x}^{\infty}} \leq C\left\|u_{0}\right\|_{H_{0}^{s}}, \quad s>1 / 2
$$


This inequality together with the trivial one

$$
\left\|\nabla|u|^{4} u\right\|_{L^{2}} \leq c\|u\|_{L^{\infty}}^{4}\|\nabla u\|_{L^{2}}
$$

allows us to do a fixed point argument in $L_{t}^{4} L_{x}^{\infty} \cap L_{t}^{\infty} H_{0}^{1}$, to solve locally in time

$$
u(t)=\mathrm{e}^{\mathrm{i} t \Delta_{\Omega}} u_{0}+\mathrm{i} \int_{0}^{t} \mathrm{e}^{\mathrm{i}(t-\tau) \Delta_{\Omega}}|u|^{4} u(\tau) d \tau .
$$

On the other hand, given any interval $I$ of time where the solution exists we know from Proposition 2 that $\|u\|_{L_{I}^{4} L_{x}^{8}}$ is finite and bounded by $E^{1 / 8} M^{3 / 8}$. Hence given any $\epsilon>0$ there is a finite number of disjoint intervals $I_{1}, \ldots, I_{N}$ such that $\cup_{j=1}^{N} I_{j}=I$ with $N=N(\epsilon)$ and

$$
\|u\|_{L_{I_{j}}^{4} L_{x}^{\infty}}^{4}=\epsilon, \quad j<N(\epsilon), \quad\|u\|_{L_{I_{j}}^{4} L_{x}^{\infty}}^{4} \leq \epsilon, \quad j=N(\epsilon) .
$$

Hence,

$$
N\left(\epsilon \leq C E^{1 / 2} M^{3 / 2} \epsilon^{-1} .\right.
$$

Assume $I_{j}=\left[t_{j}, t_{j+1}\right)$ so that

$$
\left\|u\left(t_{j}\right)\right\|_{H_{0}^{1}} \leq E^{1 / 2}
$$

and call

$$
\sigma(t)=\int_{t_{j}}^{t}\|u\|_{L_{x}^{\infty}}^{4} d t
$$

Then $\sigma(t)$ is a continuous function with $\sigma\left(t_{j}\right)=0$. Moreover for $t_{j} \leq t<t_{j+1}$

$$
\left\|D^{3 / 4} u\right\|_{L_{\left[t_{j}, t\right]}^{4} L_{x}^{8}} \leq C E^{1 / 2}\left(1+\|u\|_{L_{\left[t_{j}, t\right]}^{4} L_{x}^{\infty}}^{4}\right) .
$$

Hence,

$$
\begin{aligned}
\sigma(t) & \leq\|u\|_{L_{\left[t_{j}, t\right]}^{4} L_{x}^{8}}^{8 / 3}\left(C E^{1 / 2}(1+\sigma(t))\right)^{4 / 3} \\
& \leq C \epsilon^{2 / 3} E^{2 / 3}\left(1+\sigma(t)^{4 / 3}\right)
\end{aligned}
$$

and taking $\epsilon E$ small enough, (i.e., $\epsilon=(C E)^{-1}$ ) we conclude that $\sigma\left(t_{j+1}\right)$ remains bounded by a universal constant independent of $I$. Therefore, the solution is global and

$$
\|u\|_{L_{\mathbb{R}}^{4} L_{x}^{\infty}}^{4} \leq C N \leq C E^{3 / 2} M^{3 / 2}
$$

Finally, defining

$$
u_{+}=u_{0}+\int_{0}^{\infty} \mathrm{e}^{\mathrm{i} \tau \Delta_{\Omega}}|u|^{4} u(\tau) d \tau
$$

we obtain (1.7).

For the general case $p>5$, we need a bound for $\|u\|_{L_{t}^{p-1} L_{x}^{\infty}}$. Call $q=p-1$. Then for $\theta=4 / q$, we have

$$
\|u\|_{L_{t}^{q} L_{x}^{2 q}}^{q} \leq C \int_{0}^{+\infty}\left(\|u\|_{L_{x}^{8}}^{\theta}\|u\|_{H_{0}^{1}}^{1-\theta}\right)^{q} d t \leq C M^{3 / 2} E^{\frac{q}{2}-\frac{3}{2}} .
$$


Hence,

$$
\left\|\mathrm{e}^{i t \Delta_{\Omega}} u_{0}\right\|_{L_{t}^{q} L_{x}^{2 q}}^{q} \leq C M^{3 / 2 q} E^{\frac{1}{2}\left(1-\frac{3}{q}\right)} .
$$

Finally, it is enough to use instead of (2.8) the following inequality:

$$
\|f\|_{L^{\infty}\left(\mathbb{R}^{2}\right)} \leq C\|f\|_{L^{2 q(}\left(\mathbb{R}^{2}\right)}^{2 / 3}\left\|D^{3 / q} f\right\|_{L^{2 q}\left(\mathbb{R}^{2}\right)}^{1 / 3}, \quad q \geq 3 .
$$

The rest of the proof works similarly.

Remark 5. Notice how, in the previous argument, one is combining a Strichartz estimate of type $L_{t}^{r} L_{x}^{\infty}$ with the $H_{0}^{1}$ norm to control the source term $|u|^{p-1} u$ in $L_{t}^{1}\left(H_{0}^{1}\right)$, and this in turn yields $r=p-1$. Given that all available Strichartz estimates on domains are suboptimal with respect to regularity - there is a loss in the Sobolev embedding scale when compared to $\mathbb{R}^{2}$ - we cannot hope to use e.g., dual Strichartz pairs to allow for higher time integrability. As such, dealing with a given $p$ requires the availability of Strichartz estimates with $r=p-1$. In light of ongoing development on linear dispersion on domains, we may hope (being optimistic) for $r>12 / 5$, which is still not the $\mathbb{R}^{2}$ range, and new insights on the linear theory will be needed to match it.

\section{References}

[1] M.D. Blair, H.F. Smith and C.D. Sogge, Strichartz estimates and the nonlinear Schrödinger equation on manifolds with boundary, Math. Ann. 354(4) (2012), 1397-1430.

[2] J. Colliander, M. Grillakis and N. Tzirakis, Tensor products and correlation estimates with applications to nonlinear Schrödinger equations, Commun. Pure Appl. Math. 62(7) (2009), 920-968.

[3] J. Colliander, J. Holmer, M. Visan and X. Zhang, Global existence and scattering for rough solutions to generalized nonlinear Schrödinger equations on $\mathbb{R}$, Commun. Pure Appl. Anal. $\mathbf{7}(3)$ (2008), 467-489.

[4] J. Colliander, M. Keel, G. Staffilani, H. Takaoka and T. Tao, Global existence and scattering for rough solutions of a nonlinear Schrödinger equation on $\mathbb{R}^{3}$, Commun. Pure Appl. Math. $\mathbf{5 7}(8)$ (2004), 987-1014.

[5] - Global well-posedness and scattering for the energy-critical nonlinear Schrödinger equation in $\mathbb{R}^{3}$, Ann. Math. (2) 167(3) (2008), 767-865.

[6] J. Ginibre and G. Velo, Scattering theory in the energy space for a class of nonlinear Schrödinger equations, J. Math. Pures Appl. (9) 64(4) (1985), 363-401.

[7] — Quadratic Morawetz inequalities and asymptotic completeness in the energy space for nonlinear Schrödinger and Hartree equations, Quart. Appl. Math. 68(1) (2010), 113-134.

[8] O. Ivanovici and F. Planchon, Square function and heat flow estimates on domains (2008). arXiv:math/0812.2733.

[9] J.E. Lin and W.A. Strauss, Decay and scattering of solutions of a nonlinear Schrödinger equation, J. Funct. Anal. 30(2) (1978), 245-263.

[10] K. Nakanishi, Energy scattering for nonlinear Klein-Gordon and Schrödinger equations in spatial dimensions 1 and 2, J. Funct. Anal. 169(1) (1999), 201-225.

[11] F. Planchon and L. Vega, Bilinear virial identities and applications, Ann. Sci. Éc. Norm. Supér. (4) 42(2) (2009), 261-290.

Laboratoire J. A. Dieudonné, UMR 7351, Université de Nice Sophia-Antipolis, ParC Valrose, 06108, Nice Cedex 02, France and Institut universitaire de France

E-mail address: fabrice.planchon@unice.fr

Universidad del País Vasco, Departamento deMatemáticas, Apartado 644, 48080, BILBAo, SPAIN

E-mail address: luis.vega@ehu.es 
